\title{
A functional genetic screen identifies the Mediator complex as essential for SSX2-induced senescence
}

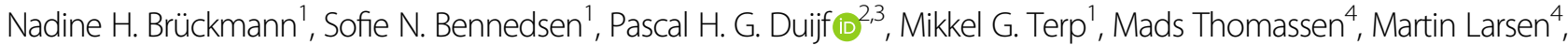

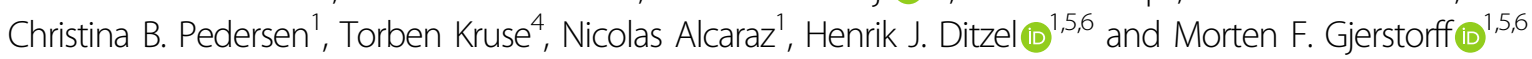

\begin{abstract}
The senescence response to oncogenes is believed to be a barrier to oncogenic transformation in premalignant lesions, and describing the mechanisms by which tumor cells evade this response is important for early diagnosis and treatment. The male germ cell-associated protein SSX2 is ectopically expressed in many types of cancer and is functionally involved in regulating chromatin structure and supporting cell proliferation. Similar to many wellcharacterized oncogenes, SSX2 has the ability to induce senescence in cells. In this study, we performed a functional genetic screen to identify proteins implicated in SSX2-induced senescence and identified several subunits of the Mediator complex, which is central in regulating RNA polymerase-mediated transcription. Further experiments showed that reduced levels of MED1, MED4, and MED14 perturbed the development of senescence in SSX2expressing cells. In contrast, knockdown of MED1 did not prevent development of B-Raf- and Epirubicin-induced senescence, suggesting that Mediator may be specifically linked to the cellular functions of SSX2 that may lead to development of senescence or be central in a SSX2-specific senescence response. Indeed, immunostaining of melanoma tumors, which often express SSX proteins, exhibited altered levels of MED1 compared to benign nevi. Similarly, RNA-seq analysis suggested that MED1, MED4, and MED14 were downregulated in some tumors, while upregulated in others. In conclusion, our study reveals the Mediator complex as essential for SSX2-induced senescence and suggests that changes in Mediator activity could be instrumental for tumorigenesis.
\end{abstract}

\section{Introduction}

Cellular senescence is an aging-related growth arrest of cells that contributes to the pathology of many diseases of the elderly, such as diabetes, cardiovascular disease, and cancer $^{1,2}$. Senescence was first described as a response to cell division-related telomere erosion in human diploid cells (i.e., replicative senescence), but over the years different types of cellular stress have been demonstrated to induce senescence, including DNA damage, oxidative stress, oncogene activation, and certain chemotherapeutic

\footnotetext{
Correspondence: Morten F. Gjerstorff (mgjerstorff@health.sdu.dk)

1 Department of Cancer and Inflammation Research, Institute for Molecular Medicine, University of Southern Denmark, Odense, Denmark

${ }^{2}$ Institute of Health and Biomedical Innovation, Faculty of Health, School of Biomedical Sciences, Queensland University of Technology, Brisbane, QLD, Australia

Full list of author information is available at the end of the article. Edited by A. Stephanou
}

drugs $^{1,2}$. Although much has been learned about the initiating factors of senescence and the phenotype of senescent cells, the molecular basis of the senescence response is still largely uncharacterized. Since the economic and social burdens associated with demographic shifts in many countries have prompted the need for better diagnostics and treatment of age-related diseases, we need a better understanding of the senescence response.

In cancer, senescence may act as a powerful barrier to cellular transformation by blocking the effects of activated oncogenes. This is supported by in vivo observations of nevi, which invariably express activated oncogenes together with classical senescence-associated markers (e.g., senescence-associated beta-galactosidase activity and p16 activation $)^{3-7}$. Furthermore, nevi show no signs of telomere attrition, suggesting that the senescence response is driven by oncogene expression rather than loss of

\section{(c) The Author(s) 2019}

(c) (i) Open Access This article is licensed under a Creative Commons Attribution 4.0 International License, which permits use, sharing, adaptation, distribution and reproduction in any medium or format, as long as you give appropriate credit to the original author(s) and the source, provide a link to the Creative Commons license, and indicate if changes were made. The images or other third party material in this article are included in the article's Creative Commons license, unless indicated otherwise in a credit line to the material. If material is not included in the article's Creative Commons license and your intended use is not permitted by statutory regulation or exceeds the permitted use, you will need to obtain permission directly from the copyright holder. To view a copy of this license, visit http://creativecommons.org/licenses/by/4.0/. 
replicative potential ${ }^{7}$. Oncogene-induced senescence has been studied in several in vitro models with strong oncogenes such as Ras, B-Raf, Cyclin E, and Myc, which suggest that replication stress may be instrumental for development of this phenotype ${ }^{8,9}$.

We recently demonstrated that SSX2 induces senescence in different types of cells, as determined by classical senescence features, including enlargement of the cytoplasm, cell growth arrest, enhanced B-galactosidase activity, and DNA double strand breaks ${ }^{10}$. SSX proteins are germ cell-specific proteins that are expressed at the spermatogonial stage of spermatogenesis ${ }^{11}$. They are also ectopically expressed in many types of tumors, such as $40 \%$ of melanomas and up to $65 \%$ of breast cancers ${ }^{12,13}$. The SSX family comprise nine highly identical members, which are most likely redundant in their cellular functions $^{14}$. SSX proteins belong to the cancer/testis (CT) antigen group of tumor antigens, and T-cell and antibody responses have been detected in cancer patients ${ }^{15}$. The role of SSX proteins in tumor formation and progression remains largely elusive. We have demonstrated that knockdown of SSX genes in melanoma cancer cells significantly reduces cellular proliferation ${ }^{10}$ and SSX proteins were shown to activate several important mitogenic pathways, such as MAPK and $\mathrm{Wnt}^{16}$. The role of SSX proteins in supporting cell proliferation and their ability to induce senescence suggest that these $\mathrm{CT}$ antigens may have oncogenic potential. SSX proteins are DNA-binding factors that regulate chromatin structure and Polycomb function $^{14,15,17,18}$. They contain an SSX repression (SSXRD) domain and a Kruppel-associated box (KRAB), which seem to be important for chromatin-binding and -rearrangement, respectively ${ }^{14}$. However, the specific mechanism by which SSX proteins regulate chromatin structure remains unknown.

In this study, we performed a functional genetic screen to identify factors essential for SSX2-induced senescence in order to further understand the molecular functions of SSX2 and the cellular response to SSX2. The results showed that several subunits of the Mediator complex are important for SSX2-induced senescence. The Mediator complex is directly implicated in RNA polymerase IImediated transcription, and its main function is to transduce signals from the transcription activators bound to enhancer regions to the transcription machinery ${ }^{19}$. Thus, Mediator is important for integration of signals from different signaling pathways that impinge on chromatin to regulate gene transcription. In addition, the Mediator complex seems to play a role in chromatin structure and the spatial positioning of chromatin in the nucleus. It has been demonstrated that Mediator interacts with the SWI/SNF and CHD1 chromatin remodeling complexes ${ }^{20-22}$ and is important for heterochromatin formation $^{23}$. Mediator is also important for the formation and stabilization of chromatin loops $\mathrm{s}^{24-26}$ and for regulation of gene expression by association with the nuclear pore complex ${ }^{27}$. The human Mediator complex generally consists of 26 subunits, but the complex seems highly flexible in structure ${ }^{28}$. Several studies have indicated that subsets of endogenous Mediator complexes may lack specific subunits and therefore acquire different functions. For instance, the thyroid hormone receptor binds the complex through MED1, and MED1 knockout cells have impaired activation of hormone receptor target genes ${ }^{29}$, but Mediator complexes lacking MED1 could integrate signals from transcription factors that bind other subunits. In addition, the Mediator complex associates with additional factors, such as the four subunit CDK8 module, which dramatically alters its structure and function ${ }^{28}$. These results suggest that the transcriptional integration of different signaling events and associated transcription factors rely on specific components and possible compositions of the Mediator complex. In accordance, Mediator complexes with a simplified subunit composition have been identified in differentiated cells ${ }^{30}$. Mediator subunits have been shown to exhibit cancer-specific transcription profiles that indicate disease-related shifts in Mediator complex composition ${ }^{31}$. Furthermore, different subunits have been implicated in cancer development or progression ${ }^{32}$.

As described above, the Mediator complex is important in multiple cellular functions and there is growing evidence for a role in cancer. In this study, we describe for the first time a role for Mediator in SSX2-mediated senescence.

\section{Results}

A functional genetic screen identified potential mediators of SSX2-induced senescence

Functional genetic screening using shRNA libraries is a powerful tool for identification of genes essential for specific cellular phenotypes. We applied this technique to a previously established model ${ }^{10}$, wherein MFC7 cells with ectopic expression of SSX2 (Fig. 1a, b) undergo a senescence response, evident by loss of proliferation, enhanced beta-galactosidase activity, and increased cell size (Fig. 1c-e). MCF7 cells were transduced with lentivirus carrying an shRNA library targeting 4922 human genes with 4-6 shRNAs per gene (Fig. 1f). Since SSX proteins are DNA-binding factors with a role in structural regulation of chromatin and might induce senescence through a chromatin-associated mechanism, we selected a library constructed by Cellecta that was focused towards genes encoding DNA binding molecules. Following lentiviral infection with shRNA-encoding plasmid constructs, the cells were treated with doxycycline to induce SSX2 expression. Subsequently, the cells were cultured for 21 days to allow senescence development (approximately 7 days) (Fig. 1f) and outgrowth of 


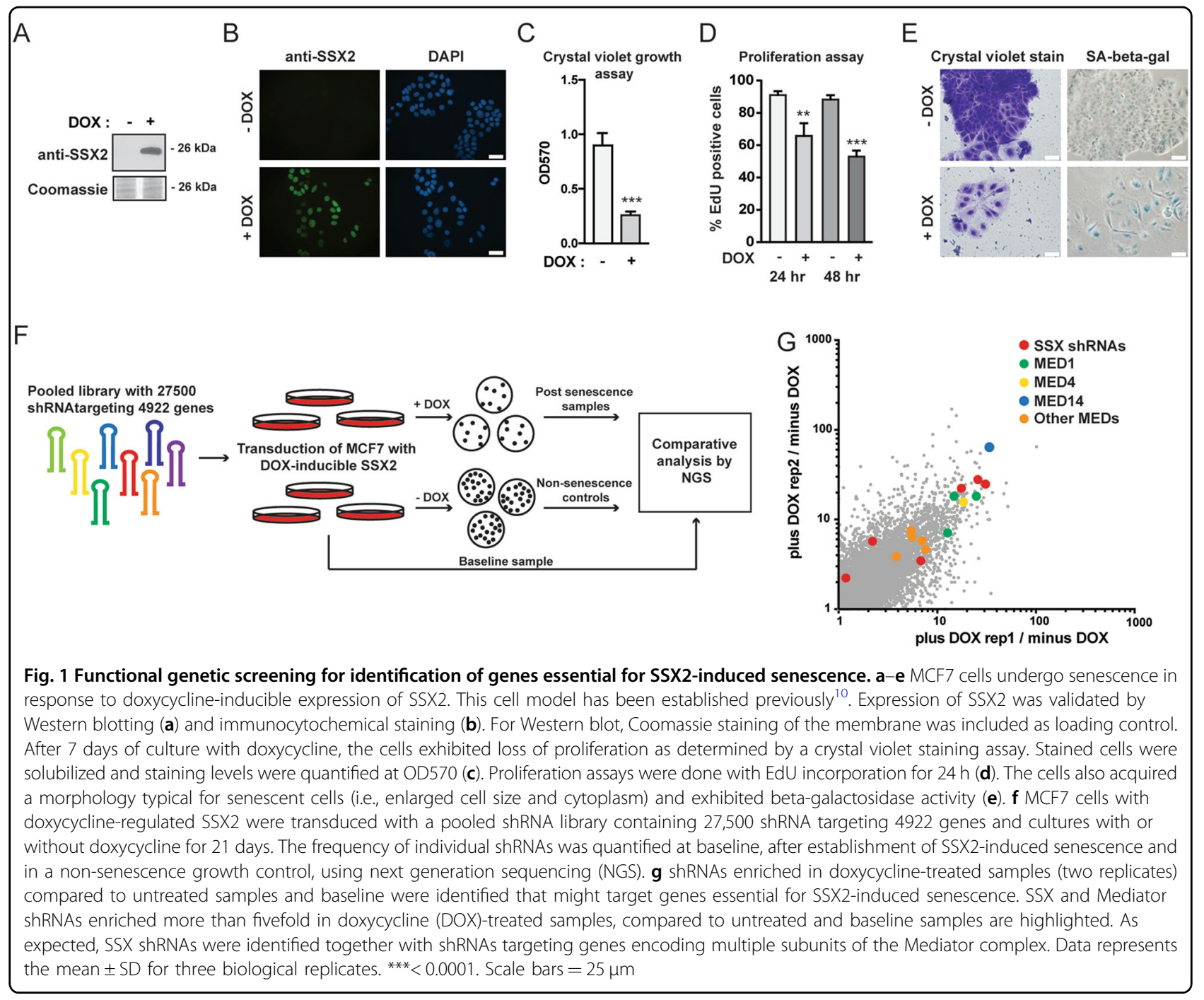

cell clones that did not undergo senescence-associated cell growth arrest due to shRNA-mediated knockdown of specific genes. Cells without SSX2 expression were cultured in parallel to control for genes that affect MCF7 cell growth in general. Massive parallel sequencing of the baseline sample (MCF7 cells infected with the library), post-senescence samples and non-senescence control samples in two replicates identified a list of shRNAs enriched in post-senescence samples that might target genes essential for the development of SSX2-induced senescence (Fig. 1g). There was a high concordance between the two replicates (Fig. 1g). Eleven genes that were targeted by at least two shRNAs enriched more than fivefold in the post-senescence samples, compared to baseline samples and the non-senescence samples, were considered potential mediators of SSX-induced senescence (Table S1). The presence of several shRNAs targeting SSX2 and homologous SSX genes was indicative of a successful approach (Fig. 1g).

\section{Mediator complex subunits are essential for SSX2-induced senescence}

Among the 11 genes identified, the most prominent were subunits of the Mediator complex (Fig. 1f; Supplementary Tables 1 and 2). Mediator complex subunits 1 and 4 (MED1 and MED4) were represented by three and two shRNAs, respectively, but single shRNAs targeting MED9, MED14, MED21, MED22, MED23, and MED26 were also highly enriched in post senescence samples (Table S2). This strongly suggested that the Mediator complex is important for SSX2-induced senescence. To validate this, we targeted the expression of MED1, MED4, and MED14 by novel sets of shRNAs in a single geneknockdown format. Despite testing many different shRNAs targeting MED1, MED4, and MED14, a general knockdown efficiency of only approximately 50-70\% (Fig. 2a) suggested that complete knockdown of these genes was not tolerated by the cells, which is in accordance with the central role of the Mediator complex in 


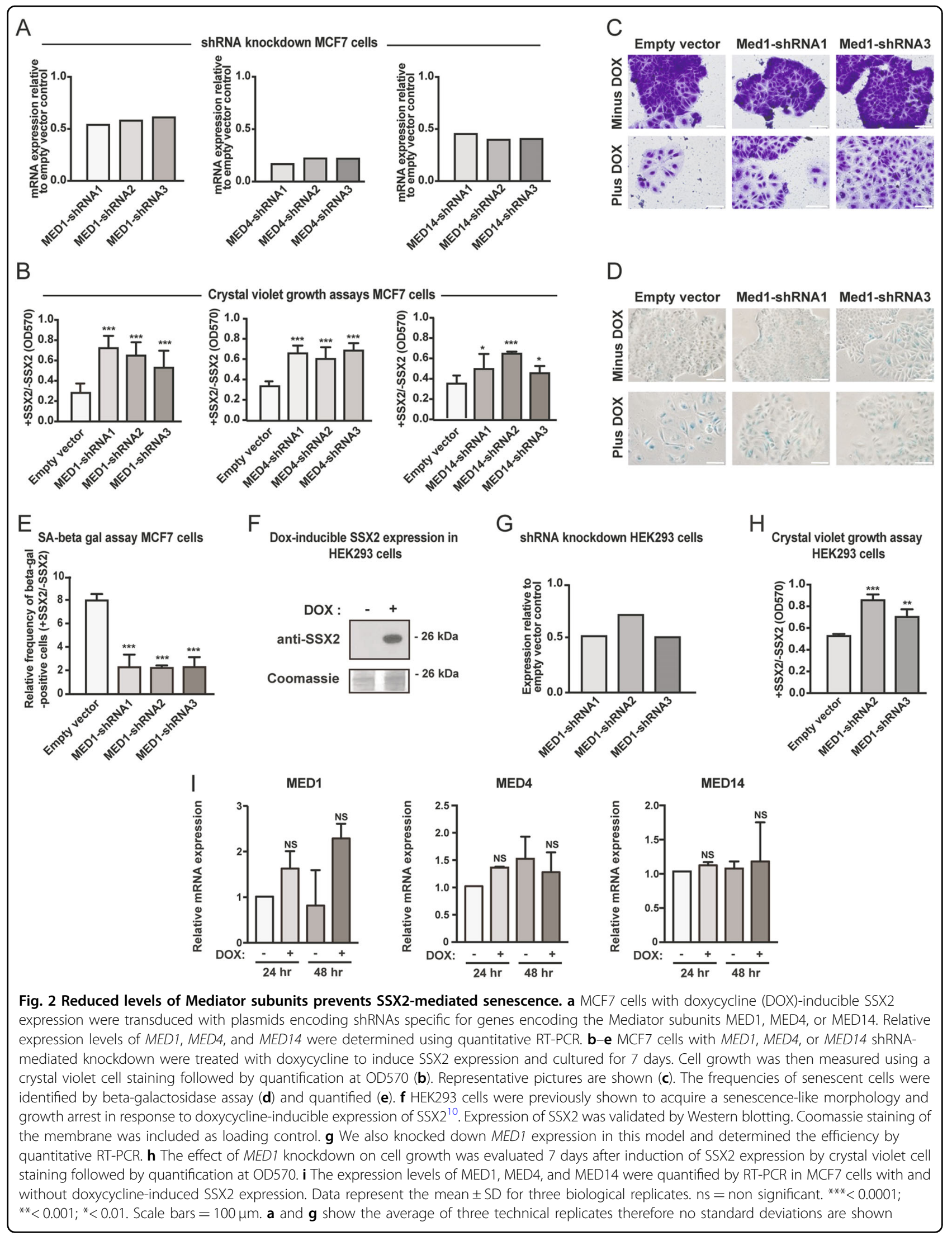


transcriptional regulation. However, reduced levels did not seem to perturb cell growth (Fig. S1). Interestingly, this reduction in expression level was sufficient for the MCF7 cells to bypass SSX2-induced, senescence-associated growth arrest and confirmed the role of MED1, MED4, and MED14 in this phenotype (Fig. 2b). In addition, a strong reduction in the frequency of enlarged (Fig. 2c) and senescence-associated, beta-galactosidase-positive cells (Fig. 2d, e) was observed following knockdown of MED1 expression. To investigate whether the role of the Mediator complex in SSX2-induced senescence was cell typespecific, we knocked down MED1 with shRNAs in the HEK293 cell line model with inducible SSX2 expression (Fig. 2f ${ }^{10}$. HEK293 cells have been demonstrated to be prone to undergo senescence ${ }^{33}$. As for MCF7 cells, the level of MED1 expression was reduced to about 50\% (Fig. 2g) and reduced levels of MED1 facilitated bypassing SSX-induced senescence-associated growth arrest (Fig. 2h), suggesting a more general role of the Mediator complex in SSX2-induced senescence. The expression levels of individual Mediator Complex subunits were not changed during SSX2-induced senescence (Fig. 2i). We also tested the ability of SSX2 to induce senescence in IMR90 fibroblast cells, which can undergo other types of senescence. SSX2 reduced the growth of the cells, but did not induce senescence characteristics such as enlarged cell size and beta-galactosidase activity (Fig. S2).

\section{MED1 is important for SSX2-mediated activation of p21 transcription}

In a previous study, we showed that SSX2-induced senescence was associated with increased levels of p53 and its downstream target, the cycline-dependent kinase inhibitor $\mathrm{p} 21^{10}$. There is strong evidence that $\mathrm{p} 53$ activity and the p53-p21 axis are major regulators of senescence in human cells ${ }^{34}$. Thus we investigated the role of p53 and two other important tumor suppressor proteins, $\mathrm{Rb}$ and PTEN, in SSX2-mediated senescence in MFC7 cells (Fig. 3). SSX2 expression was associated with increased levels of p53 and its downstream target p21 (Fig. 3a). Rb was slightly reduced a later time points ( 4 and 7 days), while PTEN remained unchanged (Fig. 3a). Knockdown of TP53, RB1, and PTEN confirmed the importance of p53 in SSX2-mediated senescence (Fig. 3b, c). Thus we investigated whether bypass of the SSX2-induced senescence response by knockdown of MED1 expression would reduce levels of p53 and p21. Indeed, we found that MCF7 cells with shRNA-mediated knockdown of MED1 levels during induction of SSX2 expression exhibited reduced levels of both p53 and p21 (Fig. 3d). Interestingly, SSX2 expression did not enhance mRNA levels of TP53 (encoding p53), suggesting that increased protein levels were achieved through protein stabilization (Fig. 3e). The role of MED1 in SSX2-induced activation of the p53-p21 axis was further indicated by reduced CDKN1A (encoding p21) expression in MCF7 cells with SSX2 expression and MED1 knockdown (Fig. 3f).

\section{Mediator complex subunits are not essential for the senescence response in general}

Having demonstrated a role for the Mediator complex in SSX2-mediated senescence in MCF7 and HEK293 cells, we investigated its role in SSX2-independent senescence using a model of $\mathrm{H}$-Ras oncogene-induced senescence in MCF7 cells ${ }^{35}$. Following overexpression of H-Ras G12V in MCF7 cells (Fig. 4a), a senescent phenotype and growth arrest similar to SSX2-expressing MCF7 cells was observed (Fig. 4b). Next, we investigated whether knockdown of MED1 expression in this model diminished senescence development in response to $\mathrm{H}$-Ras expression. Interestingly, no significant effect of MED1 knockdown on senescence-associated growth inhibition or morphological changes induced by H-Ras was observed (Fig. 4a, b), suggesting that the Mediator complex is important for the cellular response to SSX2, but not to H-Ras.

We also investigated the role of Mediator in a druginduced senescence model, where MCF7 cells undergo senescence in response to brief Epirubicin treatment (Fig. 4d-f). Knockdown of MED1 in this model did not affect the Epirubicin-induced increase in betagalactosidase activity and increase in cell size (Fig. 4d, e). Nor did it significantly reduce senescence-associated loss of proliferation, although there was a tendency towards such an effect (Fig. 4f).

These results suggest a more specific role for the Mediator complex in the molecular function exerted by SSX2 in MCF7 cells, which ultimately leads to senescence. It could also be due to mechanistic differences in the senescence response to SSX2 and H-Ras/Epirubicin, where only the former may depend on the conserved activity of the Mediator complex.

\section{Expression of Mediator subunits is deregulated in melanoma}

Our results showing that reduced expression of $M E D 1$, $M E D 4$, and MED14 prevented SSX2-mediated senescence suggests that diminished Mediator complex activity might be a compensating factor for maintaining proliferation in response to SSX expression in tumors. To this end, we investigated the potential deregulation of the Mediator complex subunits in clinical tumor samples. A direct correlation between the Mediator complex activity and SSX expression is complicated by the many different Mediator complex subunits and SSX members, and thus decreased activity of the Mediator complex in a particular tumor could be facilitated by reduced expression of any of the subunits. In agreement, there was no obvious 

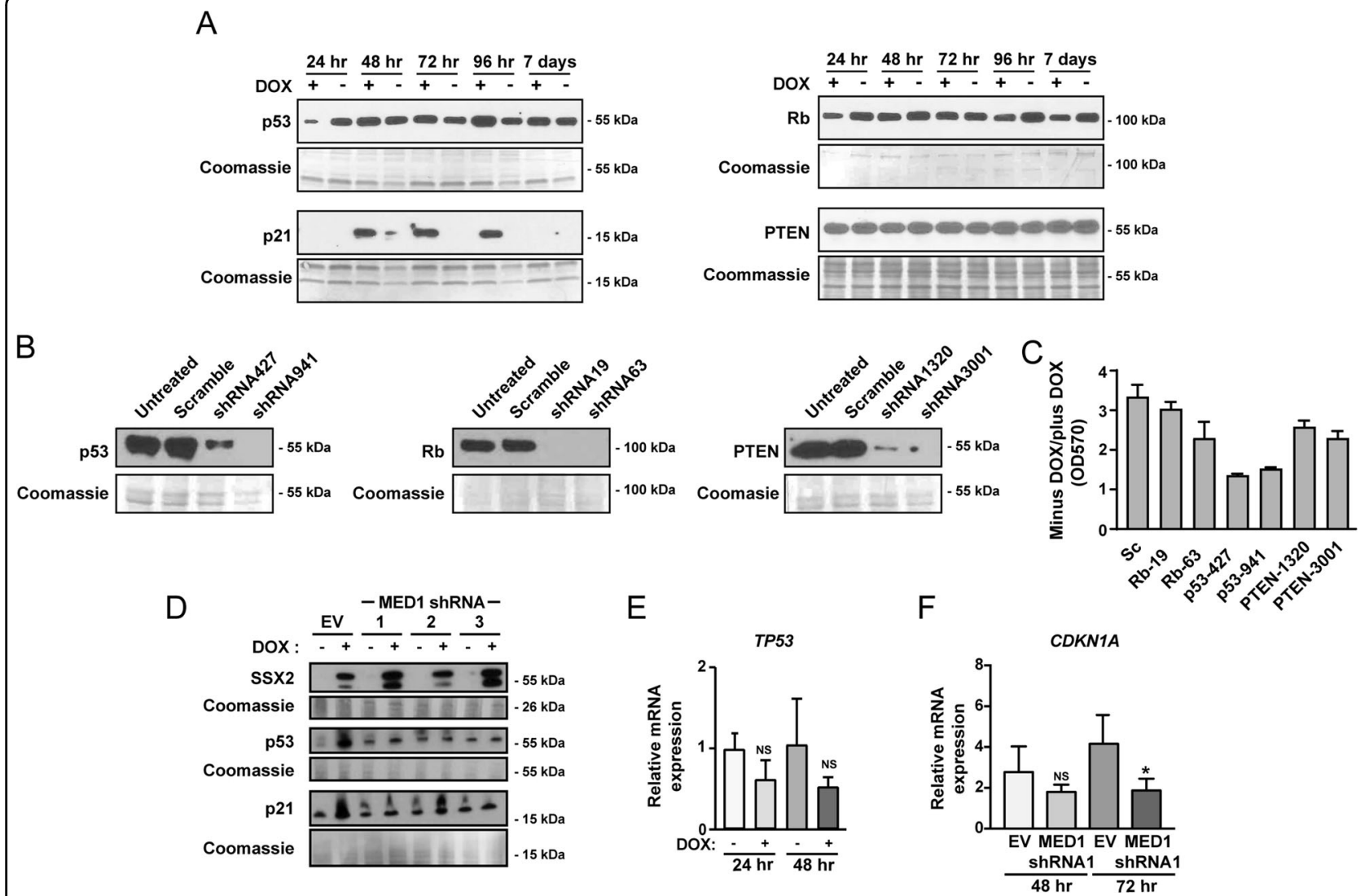

Fig. 3 The effect of MED1 in SSX2-mediated activation of p53-p21. a The SSX2-mediated induction of important tumor suppressors was investigated using Western blotting at indicated time points after addition of doxycycline to induce SSX2 expression. Coomassie staining of the membrane was included as loading control. b The expression of genes encoding p53, Rb, and PTEN was knocked down using lentiviral transductions with shRNA expression plasmids and confirmed with Western blotting. Coomassie staining of the membrane was included as loading control. c The effect of p53, Rb, and PTEN knockdown on SSX2-mediated growth arrest was investigated using crystal violet staining aften 6 days of doxycyclineinduced SSX2 expression and quantified at OD570. $\mathbf{d}$ The effect of 6 days of doxycycline-induced SSX2 expression on the levels of p53 and p21 in MCF7 cells with and without reduced MED1 expression was investigated using Western blotting. Coomassie staining of the membrane was included as loading control. e, $\mathbf{f}$ The expression levels of TP53 (e) and CDKN1A (f) were quantified by RT-PCR in MCF7 cells with and without doxycyclineinduced SSX2 expression. ${ }^{*}<0.01$

anti-correlation between expression of SSX2 and different Mediator subunits in tumors (Fig. S3). Thus, instead of a direct correlation of SSX2 and Mediator complex levels, we investigated the potential deregulation of Mediator complex subunits in various cancer types, including breast cancer and melanoma, which often express SSX2 and other SSX proteins (Fig. 5 and S4). In general, TCGA RNA-seq data showed that expression of MED1, MED4, and MED14 are not lost in tumors, but varies considerably between tumor specimens compared normal (Fig. 5). In some cancer types, the expression was reduced in tumors compared to normal tissues, but in others it was increased. In breast cancer, MED1 and MED4 expression was significantly reduced in tumors, while MED14 was increased (Fig. 5a). In melanoma, MED1 and MED14 was increased, while MED4 was reduced (Fig. 5a). Strikingly, MED1 expresssion was significantly enhanced in HER2positive breast cancers, while reduced in the basal-like subtype (Fig. 5b). MED1 and MED4 expression was generally slightly reduced with breast cancer progression (Fig. 5b), while MED14 expression was generally increased (Fig. 5b).

We also investigated the expression of MED1 using immunohistochemical staining. First, we validated the specificity of the MED1 antibody by staining a panel of normal tissues. As expected, that MED1 was detected in all tissues except the liver, and was localized predominantly in the nuclei (Fig. 6a), in accordance with data from the Human Protein Atlas (www.proteinatlas.org). Next, we investigated if MED1 expression is deregulated in association with loss of the senescence response during tumorigenesis. This was most appropriately done in melanoma, where premalignant specimens are easily accessible (i.e., nevi). Thus, we quantified MED1 levels in a panel of benign nevi $(n=39)$ and melanoma tumors $(n=74)$ using immunohistochemical staining. Melan-A 


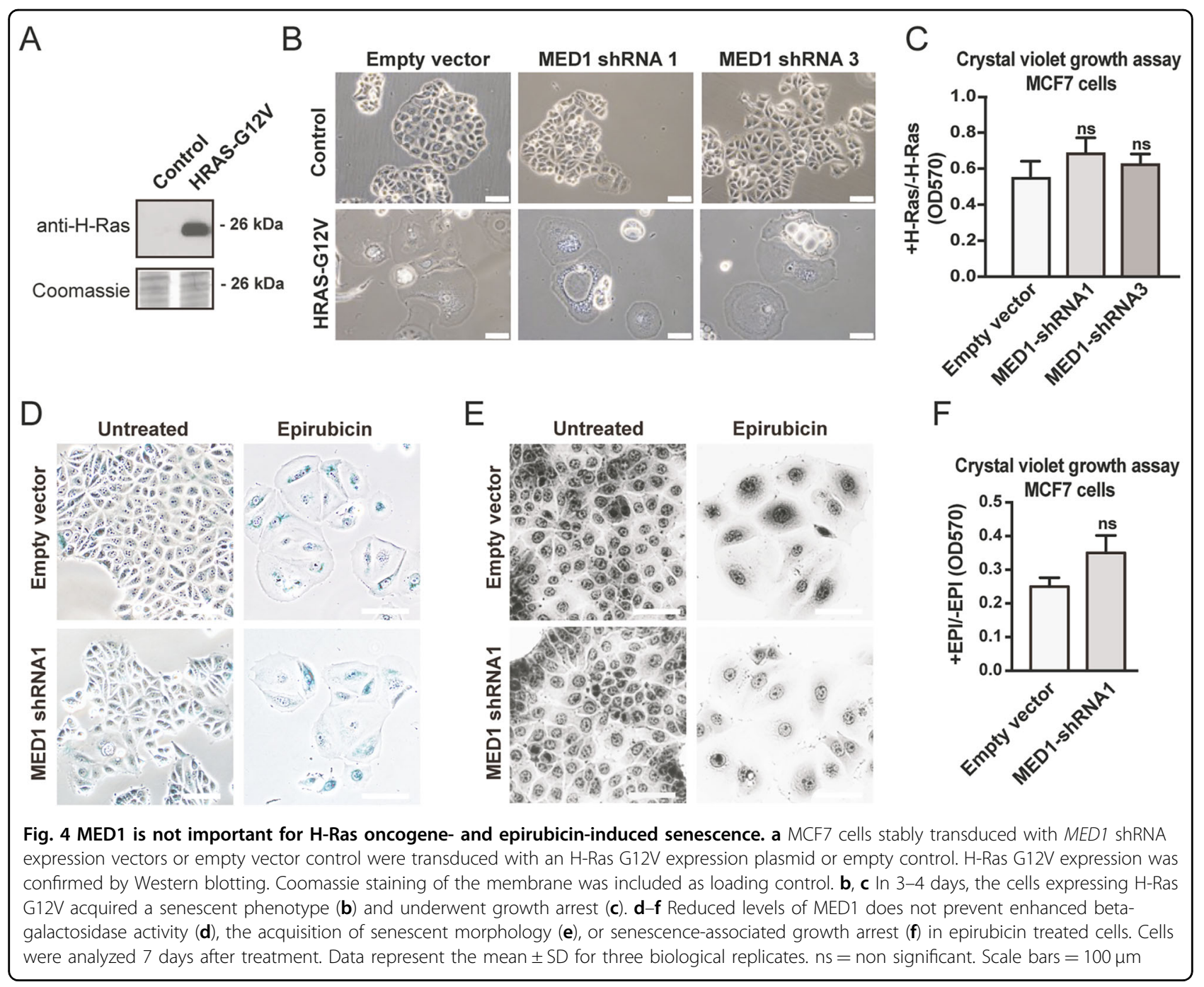

was stained on parallel tissue sections for identification of melanocytic lesions (for an example see Fig. S5). The analysis demonstrated consistent medium levels of MED1 expression in the nuclei of all melanocytic cells of benign dermal and compound nevi, whereas expression in the nuclei of melanomas was much more inconsistent, including specimens with high, medium, or low/no expression (Fig. 6b). Within both benign and malignant tumors, MED1 exhibited a relatively homogenous expression pattern (Fig. 6b). Immunohistochemical staining of MED1 showed that $31 \%$ and $27 \%$ of melanoma specimens exhibited high or low/no expression, respectively (Fig. 6c). These results were consistent with a previous study reporting that MED1 was often either upregulated or downregulated in melanoma ${ }^{36}$, which also showed that low MED1 expression correlated with a highly tumorigenic phenotype. Multiple other studies have also demonstrated that MED1 $1^{31,37-41}$ and Mediator subunits are deregulated in melanoma and other cancer types ${ }^{31,42-46}$. The observed differences in expression of different MEDs may translate into diffences in the activity of the Mediator complex to support tumorigenesis and specific phenotypes, such as SSX2 expression.

\section{Discussion}

The SSX2 protein, like well-established oncogenes such as B-Raf, Ras, Cyclin D, and Myc, supports cancer cell proliferation, but at the same time induces senescenceassociated growth arrest when ectopically expressed ${ }^{10}$. This paradox most likely reflects the need for additional alterations that prevent or bypass the senescence response. Identifying such alterations is an important step toward a better understanding of tumor initiation. In this study, we used a functional genetic screening approach to identify genes that, when reduced in expression, prevent a SSX2-induced senescence response. Interestingly, we identified several subunits of the Mediator complex as essential for this phenotype to develop. 


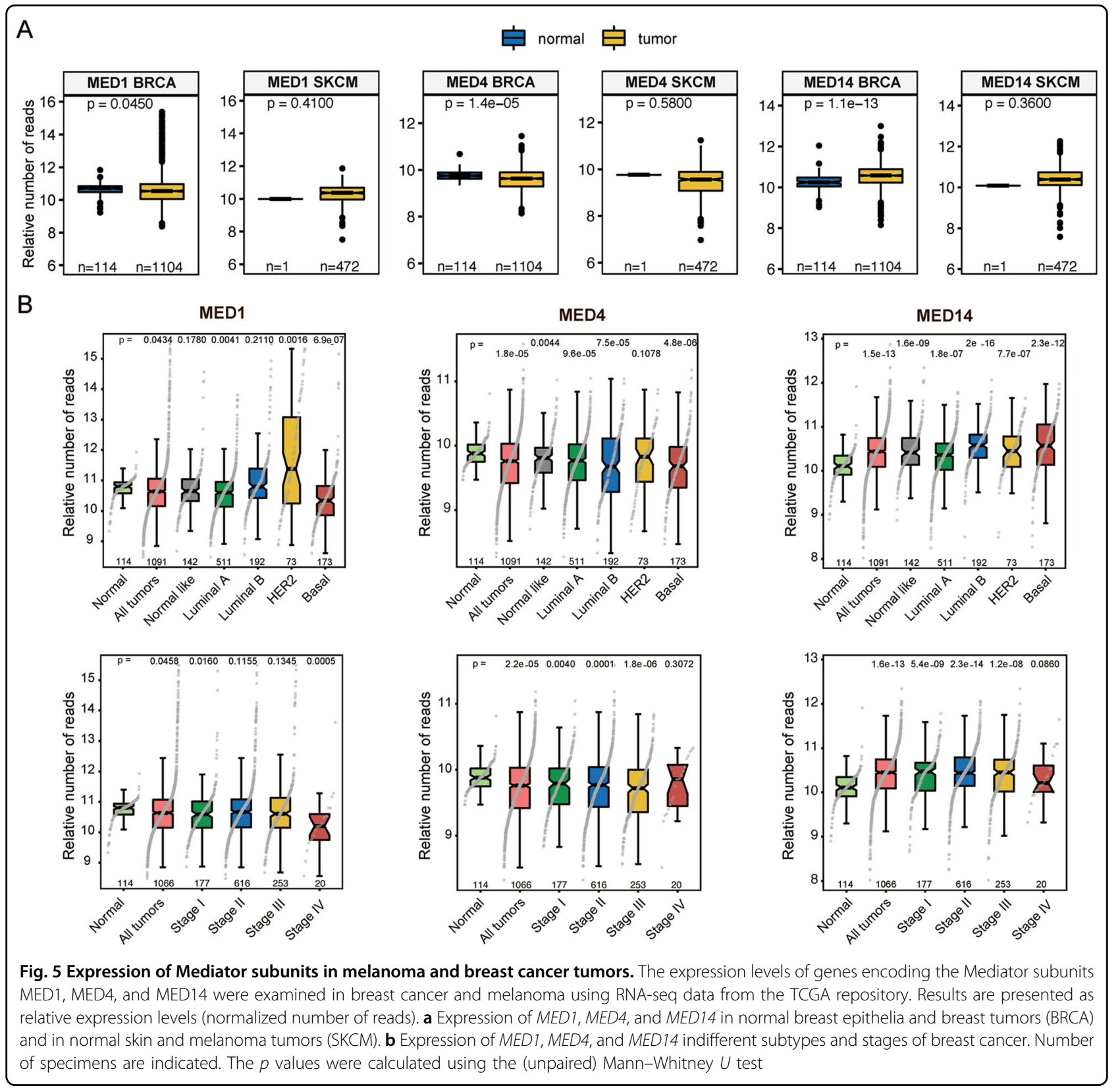

Notably, it was only possible to achieve $50-80 \%$ knockdown of MED1, MED4, and MED14, and cells with reduced expression exhibited reduced growth. This suggested that complete loss of Mediator was not tolerated by the cells, which is in accordance with other studies showing that complete knockout of Mediator subunits is embryonically lethal and impairs cell growth and cell cycle progression ${ }^{3}$, but in disagreement with another study showing that complete $M E D 1$ knockdown is indeed possible ${ }^{47}$. However, a reduction in the levels of Mediator subunits was enough to prevent SSX2-induced senescence in MCF7 and HEK293 cells. Interestingly, SSX2 did not induced a senescence response in IMR90 fibroblast cells, suggesting that that the ability of SSX2 to induce senescence is dependent on cell types or specific molecular patterns.

The Mediator complex has a multifaceted role in integrating cellular signals into changes into transcriptional reprogramming, but has not been described in association with senescence. Senescence is a complex phenotype, which, in addition to loss of proliferative signaling, also involves major changes to the chromatin regulation, metabolism and structure of the cell ${ }^{28}$. A role for Mediator in senescence development was, therefore, not unexpected. Moreover, the Mediator complex has previously been linked to the function of p53. In one study, 


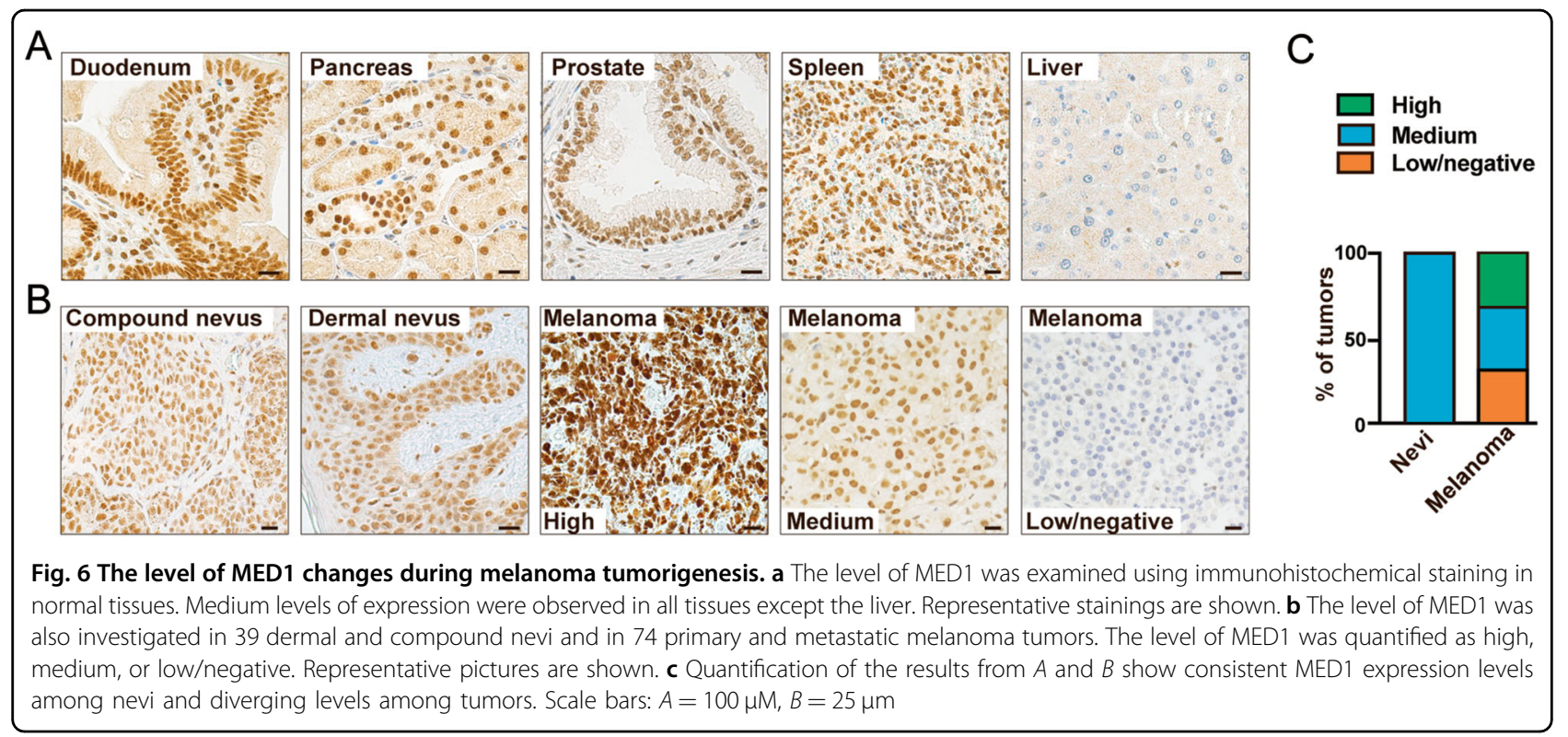

Mediator subunits, including cofactor CDK8, were shown to be recruited to the CDKN1A locus together with p53 and modulate the strength of p53-mediated transcriptional activation ${ }^{48}$. In another study, p53 was demonstrated to activate transcription by directly binding and changing the conformation of the Mediator complex ${ }^{49}$. Thus, there seems to be an intimate relationship between p53-activity and the functions of the Mediator complex. Whether interactions with the Mediator complex also stabilizes p53, as indicated here (Fig. 3a, b), remains to be determined. The prominent role of p53 in the senescence response and the direct link between the Mediator complex and p53 activity further suggested a role for the Mediator complex in senescence. However, knockdown of MED1 in other models of senescence in which MCF7 cells undergo senescence in response to the $\mathrm{H}$-Ras oncogene or Epirubicin suggested that the activity of the Mediator complex per se was not instrumental for development of senescence (Fig. 4). Our data linked the Mediator complex to the SSX-mediated senescence.

There are several posibile ways that the Mediator complex may mediate SSX2-induced senescence. The Mediator complex may be central for the senescence response to SSX2 perhaps by regulating the gene expression of central factors. This does not seem to include regulation of TP53 as this gene was not significantly induced in response to SSX2 expression. The Mediator complex may also be important for SSX2 functions, which include but may not be limited to, chromatin regulation/rearrangement. The latter is supported by our recent data demonstrating that SSX proteins bind and rearrange chromatin in cancer cells, and it is likely that the effect of the SSX2 protein on chromatin structure and gene expression is what initiates SSX2mediated senescence. Importantly, SSX proteins seem to play an antagonistic role in Polycomb group (PcG) gene repression, suggesting that ectopic expression of SSX molecules may release PcG-repressed genes ${ }^{17}$ and facilitate binding of RNA polymerase II preinitiation complex (PIC) to change the transcription landscape. In agreement with this, PcG complexes have been demonstrated to directly inhibit the assembly of the $\mathrm{PIC}^{50}$. Thus, the Mediator complex may facilitate the SSX-mediated changes in gene expression that initiates a senescence response. Another possibility is that the SSX2-mediated structural rearrangement of chromatin involves the Mediator complex, which is important for several aspects of chromatin organization.

An important implication of our results is that reduced levels of MED1, MED4, or MED14 may support specific cancer-associated alterations (e.g., SSX2 expression). This observation is interesting in the light that the expression of multiple subunits of the Mediator complex, including MED1, MED4, or MED14, is frequently dysregulated in different types of cancer.

\section{Materials and methods \\ Cells and culture conditions}

MCF7-SSX2, HEK293-SSX2 cells were grown in DMEM (Sigma Aldrich, Brondby, Denmark), 10\% fetal bovine serum (FBS) and penicillin/streptomycin, supplemented with $6 \mathrm{ng} / \mathrm{ml}$ insulin (MCF7). When relevant, cell identities according to ATCC were verified using DNA fingerprinting by short tandem repeat analysis (Cell IDTM system, Promega) and tested for mycoplasma (MycoAlert, Mycoplasma detection kit, Lonza). MCF7 
cells were were treated for two hours with $1 \mu \mathrm{M}$ Epirubicin to induce senescence. IMR90 cells were cultured in EMEM (Sigma Aldrich, Brondby, Denmark), 10\% FBS and penicillin/streptomycin. IMR90 cells were transduced with a lentiviral vector for expression of doxycyclineinducible SSX2 expression as previously described ${ }^{14}$.

\section{shRNA library preparation}

The shRNA screen was conducted using the Decipher module 3 pooled shRNA library (Cellecta Inc.), which contains 27500 shRNAs targeting 4922 human genes (i.e., 5-6 shRNAs per gene). The library was prepared as lentiviral particles by co-transfection of HEK293T cells with second-generation packaging plasmids psPAX2 and pMD2.G (Cellecta Inc.) using Lentifectin transfection reagent (Biocat, Germany). Lentiviral particles were harvested after $72 \mathrm{~h}$ and concentrated using Lenti-X concentrator (Takara Clontech).

\section{Functional genetic screening with a pooled shRNA library}

MCF7 cells with doxycycline-inducible expression of SSX2 (i.e., MCF7-SSX2) were infected with lentiviral particles carrying the pooled shRNA library in the presence of $5 \mu \mathrm{g} / \mathrm{ml}$ polybrene to obtain $40 \%$ transduced cells. Cell numbers were adjusted to maintain a library representation of $>400$. Cells were seeded in culture plates and selected with $0.2 \mu \mathrm{g} / \mathrm{ml}$ puromycin for 5 days. Cells were harvested and reseeded at a density of approximately $2.2 \times 10^{3}$ cells $/ \mathrm{cm}^{2}$ in four replicates. The remaining cells were frozen for later use as baseline samples. Two replicates were supplemented with $100 \mathrm{ng} / \mathrm{ml}$ doxycycline to induce SSX2 expression and cells were cultured for 21 days with addition of fresh media and doxycycline every three days. Next, gDNA was isolated from the baseline sample, no-doxycycline controls and doxycycline-treated cells using the Blood and cell culture maxi kit (Qiagen) according to the manufacturers recommendations and sheared into smaller fragments using a tip-sonicator set at $20 \%$ for $2 \times 10$ s. shRNA library barcodes were amplified with nested polymerase chain reaction (PCR) (see Table S3 for primers) using Titanium Taq DNA polymerase (Clontech-Takara). For the first PCR, $200 \mu \mathrm{g}$ of gDNA were used to maintain library coverage and reactions were run for 16 cycles. A fraction of the product $(1 / 80)$ was reamplified with indexing primers for 14 cycles in a second PCR. Products were purified from a 3\% agarose gel using the Nucleospin gel and PCR cleanup kit (Macherey-Nagel, Dueren, DE) and quantified with Quant-iT picogreen reagent (firma). Samples were pooled and subjected to end sequencing using a Hiseq sequencer (Illumina, San Diego, CA, USA). Sequencing data were aligned to the library of barcode identifiers using the Barcode deconvoluter software (Cellecta) and normalized to total reads. Only barcodes identified with more than 100 reads in the doxycyclinetreated samples were considered in the further analysis comparing barcode frequencies from the baseline sample, no-doxycycline controls and doxycycline-treated cells.

\section{Growth assays}

Cells were seeded in 6-well plates at a density of 5000 cells/well, and after $24 \mathrm{~h} 100 \mathrm{ng} / \mathrm{ml}$ of doxycycline was added. After approximately 10 days, plates were washed in PBS and stained with $5 \mathrm{mg} / \mathrm{ml}$ crystal violet in $25 \%$ methanol, $\mathrm{H}_{2} \mathrm{O}$ for 15 min. After 3 washes in $\mathrm{H}_{2} \mathrm{O}$, plates were air-dried and photographed or the crystal violet stain was solubilized in buffer consisting of $29.41 \mathrm{mg} / \mathrm{ml}$ citrate in $50 \%$ ethanol, $\mathrm{H}_{2} \mathrm{O}$ and quantified on a Spectramax Paradigm reader (Molecular Devices) at OD570.

\section{Senescence-associated beta-galactosidase assay}

Senescence-associated beta-galactosidase activity was measured at $\mathrm{pH} 6.0$ using a senescence beta-galactosidase staining kit (Cell Signaling) according to the manufacturer's recommendations.

\section{Western blotting}

Extracts were made from monolayers of cells with RIPA buffer, resolved by 4-20\% sodium dodecyl sulfate polyacrylamide gel electrophoresis and electroblotted onto a polyvinylidene fluoride membrane. The membrane was blocked in PBS, $0.1 \%$ Tween-20, and 5\% non-fat dry milk powder and then incubated with anti-SSX2-4 $(1: 3000)^{51}$, p53 (Santa Cruz Biotechnology, sc-126, 1:500), p21 (Santa Cruz Biotechnology, sc-53870, 1:1000), Rb (Cell Signaling, \#9309, 1:2000), PTEN (Cell Signaling, \#9188, 1:1000), or anti-H-Ras (Santa Cruz Biotechnology, sc-520, 1:1000). The blot was further stained with horseradish peroxidaseconjugated goat anti-mouse IgG (DakoCytomation Denmark A/S, Glostrup, Denmark) and developed with an ECL Western Blot kit (Amersham Biosciences, Hilleroed, Denmark, 1:100.000). All antibody incubation and washing steps were carried out in PBS, 0.1\% Tween-20.

\section{Quantitative RT-PCR}

RNA was purified from cells using RiboZol (VWR) followed by cDNA synthesis using the RevertAid Premium Reverse Transcriptase kit from Fermentas. Quantitative real-time PCR was performed using SYBR green based expression analysis (Applied Biosystems) in combination with Quantitect primers: MED1 \#QT00076356, MED4 \#QT00039158, MED14 \#QT00063490, PUM1 \#QT00029421, CDKN1A (\#QT00062090), and TP53 (\#QT00060235).

\section{shRNA knockdown}

Annealed oligoes (Table S4) were ligated into the pSico plasmid using standards methods. The shRNA plasmids 
were prepared as lentivirus by cotransfection with pMD2. G, pRSV-Rev, pMDL g/p RRE (kindly provided by the Trone Lab through Addgene, Cambridge, UK) into HEK293T cells using Lentifectin (Abmgood, Richmond, Canada). Virus was harvested from the supernatant after 2 and 3 days, filtered, precipitated with PEG and resuspended in PBS. For TP53, CDKN1A and PTEN knockdown, pLKO1 shRNA plasmids were purchased from Sigma Aldrich and prepared as lentivirus as described above.

\section{Indirect immunofluorescence}

Cells grown on coverslips were fixed in $4 \%$ formaldehyde, permeabilized in $0.2 \%$ Triton X100, PBS and blocked in $3 \%$ bovine serum albumin (BSA), PBS. Immunostaining was done with anti-SSX2/SSX3 (clone 1A4; Sigma Aldrich; 1:100) in 1\% BSA, PBS, and goat antimouse IgG $(\mathrm{H}+\mathrm{L})$ cross-adsorbed Alexa Fluor 488/568 (Thermo Fisher Scientific). Cells were mounted under cover slides with ProLong Gold Antifade with DAPI (Life Technologies) and imaging was performed with an Olympus IX73 microscope fitted with a PlanApo N 60X/ 1.42 oil objective.

\section{Immunohistochemical staining}

Tissues sections were deparaffinized and treated with $1.5 \% \mathrm{H}_{2} \mathrm{O}_{2}$ in Tris-buffered saline ( $\left.\mathrm{pH} 7.5\right)$ for $10 \mathrm{~min}$ to block endogenous peroxidase activity. Tissues were then washed in TNT buffer $(0.1 \mathrm{~m}$ Tris, $0.15 \mathrm{~m} \mathrm{NaCl}, 0.05 \%$ Tween-20, pH 7.5) and subjected to antigen retrieval with different protocols, including microwave boiling for 15 minutes in (1) T-EG buffer $(10 \mathrm{~mm}$ Tris, $0.5 \mathrm{~mm}$ EGTA, pH 9.0), (2) $10 \mathrm{~mm}$ citrate buffer (pH 6.0), or (3) Dako Target retrieval solution (Dako S1699), or proteolytic treatment using (4) $0.05 \%$ protease type XIV (pronase E, Sigma, cat. no. P5147) in TBS (pH 7.0) for 15 minutes at $37^{\circ} \mathrm{C}$ or (5) $0.4 \%$ pepsin (Sigma, Cat. No.: P7012) in $0.01 \mathrm{~m} \mathrm{HCl}$ for $20 \mathrm{~min}$ at $37^{\circ} \mathrm{C}$. Optimal atigen retrieval was achieved with microwave boiling in T-EG buffer for $15 \mathrm{~min}$. Sections were then incubated with rabbit anti-TRAP220/MED1 antibody (NB100-2574; Novus Biologicals) or mouse anti-Melan A (Clone A103; Ventana Medical Systems) diluted in antibody diluent (S2022, DAKO Cytomation, Glostrup, Denmark) for $1 \mathrm{~h}$ at room temperature. Subsequently, sections were washed with TNT, incubated with EnVision Flex/HRP+ for 30 minutes, washed again and incubated with 3,3'diaminobenzidine $(\mathrm{DAB})+$ substrate-chromogen for 10 min. Following another wash with $\mathrm{H}_{2} \mathrm{O}$, sections were counterstaining with Mayers hematoxylin before mounting in AquaTex (Merck Inc., Whitehouse Station, NJ, USA). Normal tissues analyzed for MED1 expression included: Tonsil, skin, esophagus, parotis, lung, thyroid, spleen, liver, gall bladder, colon, duodenum, muscle, testis, prostate, bladder, kidney, uterus, and placenta.

\section{Statistical testing}

Statistical testing was done using the $t$ test to compared two groups or the one-way ANOVA for comparing multiples groups (Figs. 1-4). For statistical testing of larger data sets (Fig. 5), the Mann-Whitney $U$ test was used.

\section{Acknowledgements}

We thank Ole Nielsen and Lisbet Mortensen (Department for Pathology, Odense University Hospital) for technical assistance with

immunohistochemical staining and M.K. Occhipinti for editorial assistance. This work was supported by the Velux Foundation, the Danish Cancer Society, Academy of Geriatric Cancer Research (AgeCare), and the Danish Research Council for Independent Research.

\section{Author details}

'Department of Cancer and Inflammation Research, Institute for Molecular Medicine, University of Southern Denmark, Odense, Denmark. ${ }^{2}$ Institute of Health and Biomedical Innovation, Faculty of Health, School of Biomedical Sciences, Queensland University of Technology, Brisbane, QLD, Australia. ${ }^{3}$ University of Queensland Diamantina Institute, The University of Queensland, Translational Research Institute, 37 Kent Street, Brisbane, QLD 4102, Australia. ${ }^{4}$ Department of Clinical Genetics, Odense University Hospital, Odense, Denmark. ${ }^{5}$ Department of Oncology, Odense University Hospital, Odense, Denmark. ${ }^{6}$ Academy of Geriatric Cancer Research (AgeCare), Odense University Hospital, Odense, Denmark

Conflict of interest

The authors declare that they have no conflict of interest.

\section{Publisher's note}

Springer Nature remains neutral with regard to jurisdictional claims in published maps and institutional affiliations.

Supplementary Information accompanies this paper at (https://doi.org/ 10.1038/s41419-019-2068-1).

Received: 13 June 2019 Revised: 14 October 2019 Accepted: 16 October 2019

Published online: 06 November 2019

\section{References}

1. Lopez-Otin, C., Blasco, M. A., Partridge, L., Serrano, M. \& Kroemer, G. The hallmarks of aging. Cell 153, 1194-1217 (2013).

2. Collado, M. \& Serrano, M. Senescence in tumours: evidence from mice and humans. Nat. Rev. Cancer 10, 51-57 (2010).

3. Yin, J. W. \& Wang, G. The Mediator complex: a master coordinator of transcription and cell lineage development. Development 141, 977-987 (2014).

4. Kiuru, M. et al. Improving classification of melanocytic nevi: Association of BRAF V600E expression with distinct histomorphologic features. J. Am. Acad. Dermatol. 79, 221-229 (2018).

5. Roh, M. R., Eliades, P., Gupta, S. \& Tsao, H. Genetics of melanocytic nevi. Pigment Cell Melanoma Res. 28, 661-672 (2015).

6. Pollock, P. M. et al. High frequency of BRAF mutations in nevi. Nat. Genet. 33, 19-20 (2003)

7. Michaloglou, C. et al. BRAFE600-associated senescence-like cell cycle arrest of human naevi. Nature 436, 720-724 (2005).

8. Di Micco, R. et al. Oncogene-induced senescence is a DNA damage response triggered by DNA hyper-replication. Nature 444, 638-642 (2006).

9. Macheret, M. \& Halazonetis, T. D. Intragenic origins due to short G1 phases underlie oncogene-induced DNA replication stress. Nature 555, 112-116 (2018).

10. Greve, K. B. et al. Ectopic expression of cancer/testis antigen SSX2 induces DNA damage and promotes genomic instability. Mol. Oncol. 9, 437-449 (2015).

11. Gjerstorff, M. F., Burns, J. \& Ditzel, H. J. Cancer-germline antigen vaccines and epigenetic enhancers: future strategies for cancer treatment. Expert Opin. Biol. Ther. 10, 1061-1075 (2010) 
12. Mischo, A. et al. Prospective study on the expression of cancer testis genes and antibody responses in 100 consecutive patients with primary breast cancer. Int. J. Cancer 118, 696-703 (2006).

13. Greve, K. B. et al. SSX2-4 expression in early-stage non-small cell lung cancer. Tissue Antigens 83, 344-349 (2014).

14. Traynor, S. et al. Remodeling and destabilization of chromosome 1 pericentromeric heterochromatin by SSX proteins. Nucleic Acids Res. 47, 6668-6684 (2019).

15. Gjerstorff, M. F., Andersen, M. H. \& Ditzel, H. J. Oncogenic cancer/testis antigens: prime candidates for immunotherapy. Oncotarget 6, 15772-15787 (2015).

16. D'Arcy, P., Maruwge, W., Wolahan, B., Ma, L. \& Brodin, B. Oncogenic functions of the cancer-testis antigen SSX on the proliferation, survival, and signaling pathways of cancer cells. PLOS ONE 9, e95136 (2014).

17. Gjerstorff, M. F. et al. SSX2 is a novel DNA-binding protein that antagonizes polycomb group body formation and gene repression. Nucleic Acids Res. 42, 11433-11446 (2014).

18. Bruckmann, N. H., Pedersen, C. B., Ditzel, H. J. \& Gjerstorff, M. F. Epigenetic reprogramming of pericentromeric satellite DNA in premalignant and malignant lesions. Mol. Cancer Res. 16, 417-427 (2018),

19. Jeronimo, C. \& Robert, F. The mediator complex: at the nexus of RNA polymerase II transcription. Trends Cell Biol. 27, 765-783 (2017).

20. Sharma, V. M., Li, B. \& Reese, J. C. SWI/SNF-dependent chromatin remodeling of RNR3 requires TAF(II)s and the general transcription machinery. Genes Dev. 17, 502-515 (2003).

21. Lin, J. J. et al. Mediator coordinates PIC assembly with recruitment of CHD1. Genes Dev. 25, 2198-2209 (2011).

22. Lemieux, K. \& Gaudreau, L. Targeting of Swi/Snf to the yeast GAL1 UAS G requires the Mediator, TAF IIs, and RNA polymerase II. EMBO J. 23, 4040-4050 (2004).

23. Oya, E. et al. Mediator directs co-transcriptional heterochromatin assembly by RNA interference-dependent and -independent pathways. PLOS Genet. 9, e1003677 (2013).

24. Kagey, M. H. et al. Mediator and cohesin connect gene expression and chromatin architecture. Nature 467, 430-435 (2010).

25. Phillips-Cremins, J. E. et al. Architectural protein subclasses shape 3D organization of genomes during lineage commitment. Cell 153, 1281-1295 (2013).

26. Muto, A. et al. Nipbl and mediator cooperatively regulate gene expression to control limb development. PLoS Genet. 10, e1004671 (2014).

27. Schneider, M. et al. The nuclear pore-associated TREX-2 complex employs mediator to regulate gene expression. Cell 162, 1016-1028 (2015)

28. Allen, B. L. \& Taatjes, D. J. The Mediator complex: a central integrator of transcription. Nat. Rev. Mol. Cell Biol. 16, 155-166 (2015).

29. Fondell, J. D., Ge, H. \& Roeder, R. G. Ligand induction of a transcriptionally active thyroid hormone receptor coactivator complex. Proc. Natl Acad. Sci. USA 93, 8329-8333 (1996).

30. Deato, M. D. et al. MyoD targets TAF3/TRF3 to activate myogenin transcription. Mol. Cell 32, 96-105 (2008).

31. Syring, I. et al. Comprehensive analysis of the transcriptional profile of the Mediator complex across human cancer types. Oncotarget 7, 23043-23055 (2016).

32. Schiano, C. et al. Involvement of Mediator complex in malignancy. Biochim. Biophys. Acta 1845, 66-83 (2014).
33. Fernandez Larrosa, P. N. et al. RAC3 more than a nuclear receptor coactivator: a key inhibitor of senescence that is downregulated in aging. Cell Death Dis. $\mathbf{6}$, e1902 (2015)

34. Rodier, F. \& Campisi, J. Four faces of cellular senescence. J. Cell Biol. 192 547-556 (2011)

35. Bihani, T., Chicas, A., Lo, C. P. \& Lin, A. W. Dissecting the senescence-like program in tumor cells activated by Ras signaling. J. Biol. Chem. 282, 2666-2675 (2007).

36. Ndong Jde, L., Jean, D., Rousselet, N. \& Frade, R. Down-regulation of the expression of RB18A/MED1, a cofactor of transcription, triggers strong tumorigenic phenotype of human melanoma cells. Int. J. Cancer 124 2597-2606 (2009)

37. Klumper, $\mathrm{N}$. et al. Mediator complex subunit MED1 protein expression is decreased during bladder cancer progression. Front. Med. (Lausanne) 4, 30 (2017).

38. Cai, Q. et al. MicroRNA-1291 mediates cell proliferation and tumorigenesis by downregulating MED1 in prostate cancer. Oncol. Lett. 17 3253-3260 (2019).

39. Zhu, Y. et al. Amplification and overexpression of peroxisome proliferatoractivated receptor binding protein (PBP/PPARBP) gene in breast cancer. Proc. Natl Acad. Sci. USA 96, 10848-10853 (1999).

40. Kim, H. J. et al. Loss of Med1/TRAP220 promotes the invasion and metastasis of human non-small-cell lung cancer cells by modulating the expression of metastasis-related genes. Cancer Lett. 321, 195-202 (2012).

41. Gade, P., Singh, A. K., Roy, S. K., Reddy, S. P. \& Kalvakolanu, D. V. Downregulation of the transcriptional mediator subunit Med1 contributes to the loss of expression of metastasis-associated dapk1 in human cancers and cancer cells. Int J. Cancer 125, 1566-1574 (2009).

42. Zhang, $\mathrm{H}$. et al. Expression of Med19 in bladder cancer tissues and its role on bladder cancer cell growth. Urol. Oncol. 30, 920-927 (2012).

43. Luoh, S. W. Amplification and expression of genes from the 17q11 approximately q12 amplicon in breast cancer cells. Cancer Genet. Cytogenet. 136, 43-47 (2002)

44. Monni, O. et al. Comprehensive copy number and gene expression profiling of the 17q23 amplicon in human breast cancer. Proc. Natl Acad. Sci. USA 98, 5711-5716 (2001)

45. Yoon, N. K. et al. Elevated MED28 expression predicts poor outcome in women with breast cancer. BMC Cancer 10, 335 (2010).

46. Ding, X. F., Huang, G. M., Shi, Y., Li, J. A. \& Fang, X. D. Med19 promotes gastric cancer progression and cellular growth. Gene 504, 262-267 (2012).

47. Cui, J. et al. Cross-talk between HER2 and MED1 regulates tamoxifen resistance of human breast cancer cells. Cancer Res. 72, 5625-5634 (2012).

48. Donner, A. J., Szostek, S., Hoover, J. M. \& Espinosa, J. M. CDK8 is a stimulus-specific positive coregulator of p53 target genes. Mol. Cell 27 121-133 (2007).

49. Meyer, K. D., Lin, S. C., Bernecky, C., Gao, Y. \& Taatjes, D. J. p53 activates transcription by directing structural shifts in Mediator. Nat. Struct. Mol. Biol. 17 753-760 (2010).

50. Lehmann, L. et al. Polycomb repressive complex 1 (PRC1) disassembles RNA polymerase II preinitiation complexes. J. Biol. Chem. 287, 35784-35794 (2012).

51. dos Santos, N. R. et al. Heterogeneous expression of the SSX cancer/testis antigens in human melanoma lesions and cell lines. Cancer Res. 60 1654-1662 (2000). 